\title{
A LOCAL CHARACTERISATION OF SERIAL SUBGROUPS
}

\author{
by J. A. HULSE \\ (Received 22nd February 1978)
}

\section{Introduction}

In (2) Hickin and Phillips establish various results connecting the ideas of local systems and serial subgroups in group theory. In (4) Petty shows that if a group has a local factor system of $\mathfrak{X}$-groups, then it may be embedded in an ultraproduct of $\mathfrak{X}$-groups and he uses this result to extend some local theorems which were originally due to Mal'cev (3) and at the same time providing an elementary proof. We shall show that Petty's method may be used to prove results about seriality and local systems.

We recall that a subgroup $H$ of a group $G$ is serial in $G$, denoted by $H \operatorname{ser} G$, if there exists a totally ordered set $\Sigma$ and a set

$$
\left\{\left(\Lambda_{\sigma}, V_{\sigma}\right) \mid \sigma \in \Sigma\right\}
$$

such that:

(a) $H \leqslant V_{\sigma} \vDash \Lambda_{\sigma} \leqslant G$ for all $\sigma \in \Sigma$;

(b) $\Lambda_{\tau} \leqslant V_{\sigma}$ if $\tau<\sigma$;

(c) $G \backslash H=\cup\left\{\Lambda_{\sigma} \backslash V_{\sigma} \mid \sigma \in \Sigma\right\}$.

Our first result will show that seriality is preserved by taking reduced direct products. We note that in general seriality is not preserved under homomorphic images and so our result does not follow immediately from the elementary fact that seriality is preserved by taking Cartesian products.

If $\left(G_{\alpha} \mid \alpha \in A\right)$ is a family of groups, we denote their Cartesian product by $\mathrm{Cr}_{\alpha \in A} G_{\alpha}$, that is the group of all maps

$$
x: A \rightarrow \cup\left\{G_{a} \mid\left\{G_{\alpha} \mid \alpha \in A\right\}\right.
$$

such that $\alpha x \in G_{\alpha}$ for all $\alpha \in A$ together with pointwise multiplication. If $H_{\alpha} \leqslant G_{\alpha}$ for each $\alpha \in A$ we identify $\mathrm{Cr}_{\alpha \in A} H_{\alpha}$ with the subgroup of $C=\mathrm{Cr}_{\alpha \in A} G_{\alpha}$ comprising all $x \in C$ such that $\alpha x \in H_{\alpha}$ for all $\alpha \in A$.

Theorem A. Let $A$ be a non-empty set, let $\mathscr{F}$ be any filter on $A$, and for each $\alpha \in A$ let $H_{\alpha}$ be a serial subgroup of a group $G_{\alpha}$. Suppose that $C=\mathrm{Cr}_{\alpha \in A} G_{\alpha}$, $\mathrm{D}=\mathrm{Cr}_{\alpha \in A} H_{\alpha}$ and

$$
N_{\mathscr{F}}=\{x \in C \mid I(x) \in \mathscr{F}\}
$$

where

$$
I(x)=\{\alpha \in A \mid \alpha x=1\}
$$


Then $N_{\mathscr{F}} \leqslant C$ and

\section{$D N_{\mathscr{F}} \operatorname{ser} C$.}

We remark here that the fact that $N_{\oiint} \leqslant C$ is well known and the group $C / N_{\mathscr{F}}$ is called the reduced direct product of $\left(G_{\alpha} \mid \alpha \in A\right)$ deteri.:Ined by $\mathscr{F}$. If $\mathscr{F}$ is an ultrafilter on $A$, then $C / N_{F}$ is called an ultraproduct, see (1), p. 179.

If $H$ is a subgroup of a group $G$, then we call a set of pairs of subgroups of $G$, $\left\{\left(H_{\alpha}, G_{\alpha}\right) \mid \alpha \in A\right\}$, a local system for $(H, G)$ if $H_{\alpha} \leqslant H \cap G_{\alpha}$ for all $\alpha \in A$ and for all finite subsets $F$ of $G$ there exists $\alpha \in A$ such that

$$
F \subseteq\left(G_{\alpha} \mid H\right) \cup H_{\alpha}
$$

In particular we have two special cases. Firstly $G_{\alpha}=G$ for all $\alpha \in A$ and $\left\{H_{\alpha} \mid \alpha \in A\right\}$ is a local system for $H$. Secondly $H_{\alpha}=H \cap G_{\alpha}$ for all $\alpha \in A$ and $\left\{G_{\alpha} \mid \alpha \in A\right\}$ is a local system for $G$. We shall prove the following local criterion for seriality.

Theorem B. Let $H$ be a subgroup of a group $G$. If there exists a local system $\left\{\left(H_{\alpha}, G_{\alpha}\right) \mid \alpha \in A\right\}$ for $(H, G)$ such that $H_{\alpha}$ ser $G_{\alpha}$ for all $\alpha \in A$, then

\section{$H \operatorname{ser} G$.}

In the corollary below, $\mathfrak{I}$ denotes the class of all groups in which the join of any collection of serial subgroups is again serial. This corollary has previously been obtained by Hickin and Phillips in (2) by using a different method.

Corollary C. Let $H$ be a subgroup of a group $G$.

(i) If $\left\{H_{\alpha} \mid \alpha \in A\right\}$ is a local system for $H$ with $H_{\alpha}$ ser $G$ for all $\alpha \in A$, then $H \operatorname{ser} G$.

(ii) If $\left\{G_{\alpha} \mid \alpha \in A\right\}$ is a local system for $G$ with $H \cap G_{\alpha} \operatorname{ser} G_{\alpha}$ for all $\alpha \in A$, then $H \operatorname{ser} G$.

(iii) If the join of any pair of serial subgroups of $G$ is serial in $G$, then $G \in \mathfrak{\Im}$.

(iv) If $G$ has a local system of $\mathfrak{J}$-subgroups, then $G \in \mathfrak{F}$.

\section{Proofs}

For the proof of Theorem $\mathrm{A}$ we shall require an alternative definition of seriality, cf. (5) p.p. 97-98. If $H$ ser $G$, then with the above notation we define a relation $<$ on $G$ by $x<y$ if either $\{x, y\} \subseteq H$ or for some $\sigma \in \Sigma$ both $y \in \Lambda_{\sigma} \mid V_{\sigma}$ and $x \in \Lambda_{\sigma}$. Then the following facts may be easily verified:

(i) $x<y<z \Rightarrow x<z$

(ii) $x, y \in G \Rightarrow x<y$ or $y<x$;

(iii) $x<1_{G} \Leftrightarrow x \in H$;

(iv) $x<y$ and $z<y \Rightarrow x z^{-1}<y$;

(v) $y<x^{y} \Rightarrow y<x$.

Conversely let a relation $<$ on $G$ satisfy (i)-(v). An equivalence relation $\sim$ may be defined on $G$ by

$$
x \sim y \Leftrightarrow x<y \text { and } y<x .
$$

Then the quotient set $\Sigma=G / \sim$ is totally ordered by $\sigma \leqslant \tau$ if and only if $x<y$ for some 
$x \in \sigma$ and $y \in \tau$. For each $\sigma \in \Sigma$, let

$$
\Lambda_{\sigma}=\{x \in G \mid x<y \text { for some } y \in \sigma\}
$$

and

$$
V_{\sigma}=\cup\left\{\Lambda_{r} \mid \tau<\sigma\right\} \cup H .
$$

Then $\left\{\left(\Lambda_{\sigma}, V_{\sigma}\right) \mid \sigma \in \Sigma\right\}$ is a series from $H$ to $G$ and so $H$ ser $G$.

If a relation $<$ on $G$ satisfies (i)-(v), then we say that $<$ demonstrates that $H \operatorname{ser} G$.

Proof of Theorem A. If $x \in C$, let

$$
I_{D}(x)=\left\{\alpha \in A \mid \alpha x \in H_{\alpha}\right\} .
$$

If $x \in D N_{\mathscr{F}}$, then $x=y z$ for some $y \in D$ and $z \in N_{\mathscr{F}}$, so $I(z) \subseteq I_{D}(x)$. But $I(z) \in \mathscr{F}$ and so $I_{D}(x) \in \mathscr{F}$. Conversely if $I_{D}(x) \in \mathscr{F}$, define $y \in C$ by

$$
\alpha y=\left\{\begin{array}{cc}
\alpha x & \text { if } \alpha \in I_{D}(x) \\
1 & \text { if } \alpha \notin I_{D}(x)
\end{array} .\right.
$$

Then $y \in D$, but $I_{D}(x)=I\left(y^{-1} x\right)$, so $I\left(y^{-1} x\right) \in \mathscr{F}$, so $y^{-1} x \in N_{\mathscr{F}}$ and so $x \in D N_{\mathscr{F}}$. Thus $x \in D N_{\mathscr{F}}$ if and only if $I_{D}(x) \in \mathscr{F}$. Now if $\Omega$ is the set of all ultrafilters containing $\mathscr{F}$, then $\cap \Omega=\mathscr{F}$ and so

$$
D N_{\mathscr{G}}=\cap\left\{D N_{\mathscr{G}} \mid \mathscr{G} \in \Omega\right\} .
$$

Hence it is sufficient to prove the result in the case when $\mathscr{F}_{F}$ is an ultrafilter and we write $N=N_{\text {gr. }}$

Let now $<_{\alpha}$ demonstrate $H_{\alpha} \operatorname{ser} G_{\alpha}$ for each $\alpha \in A$. For each $(x, y) \in C \times C$, put

$$
T(x, y)=\left\{\alpha \in A \mid \alpha x<_{\alpha} \alpha y\right\} .
$$

Let a relation $<$ be defined on $C$ by

$$
x<y \text { if and only if } T(x, y) \in \mathscr{F} \text {. }
$$

We shall show that $<$ demonstrates $D N$ ser $C$.

Now (i) and (ii) hold since

$$
T(x, y) \cap T(y, z) \subseteq T(x, z) \text { and } A \backslash T(x, y) \subseteq T(y, x) .
$$

Also if $x \in C$, then

$$
x<1 \Leftrightarrow I_{D}(x)=T(x, 1) \in \mathscr{F} \Leftrightarrow x \in D N
$$

and so (iii) holds with $H$ replaced by $D N$. Finally (iv) and (v) hold since

$$
T(x, y) \cap T(z, y) \subseteq T\left(x z^{-1}, y\right) \text { and } T\left(y, x^{y}\right) \subseteq T(y, x) .
$$

Thus < demonstrates $D N$ ser $C$.

Proof of Theorem B. Let $C$ and $D$ be as in Theorem A. We may define a map $\delta: G \rightarrow C$ by

$$
\alpha\left(g^{\delta}\right)= \begin{cases}g & \text { if } g \in\left(G_{\alpha} \backslash H\right) \cup H_{\alpha} \\ 1 & \text { if } g \in\left(G \backslash G_{\alpha}\right) \cup\left(H \backslash H_{\alpha}\right) .\end{cases}
$$


Of course in general the map $\delta$ will not be a homorphism. However, we shall pass to $C / N$, where $N=N_{\mathscr{S}}$ for some ultrafilter $\mathscr{F}$, and show that the composite map

$$
\nu: g \mapsto\left(g^{\delta}\right) N
$$

for each $g \in G$ is a monomorphism satisfying $H^{\nu}=D N / N \cap G^{\nu}$.

For each $g \in G$, let

$$
B(g)=\left\{\alpha \in A \mid \alpha\left(g^{\delta}\right)=g\right\} .
$$

If $F$ is any finite subset of $G$, then there exists $\alpha \in A$ with $F \subseteq\left(G_{\alpha} \mid H\right) \cup H_{\alpha}$. Then $\alpha \in \cap\{B(f) \mid f \in F\}$. Thus $\{B(g) \mid g \in G\}$ has the finite intersection property and so is contained in an ultrafilter $\mathscr{F}$ on $A$. If $f, g \in G$ and

$$
x=(f g)^{\delta}\left(g^{\delta}\right)^{-1}\left(f^{\delta}\right)^{-1},
$$

then it follows that

$$
B(f g) \cap B(g) \cap B(f) \subseteq I(x) .
$$

Whence $I(x) \in \mathscr{F}$ and so $x \in N$. Thus $\nu: G \rightarrow C / N$ is a homorphism. Also if $g \in G \backslash\{1\}$, then

$$
I\left(g^{\delta}\right)=A \backslash B(g) \notin \mathscr{F}
$$

and so $g^{\delta} \notin N$. Thus $\nu: G \rightarrow C / N$ is a monomorphism.

Since $H^{\delta} \mid \subseteq D, H^{\nu} \subseteq D N / N \cap G^{\nu}$. On the other hand suppose $g \in G \backslash H$ and $g^{\nu} \in D N / N$. Then $g^{\delta} \in D N$ and so for some $x \in D x g^{\delta} \in N$ and $I\left(x g^{\delta}\right) \in \mathscr{F}$. Now

$$
\alpha\left(x g^{\delta}\right)=(\alpha x)\left[\alpha\left(g^{\delta}\right)\right]= \begin{cases}(\alpha x) g & \text { if } \alpha \in B(g) \\ \alpha x & \text { if } \alpha \notin B(g)\end{cases}
$$

But $g \notin H$ and $\alpha x \in H_{\alpha} \leqslant H$ and so $(\alpha x) g \neq 1$. Thus

$$
I(x) \cap(A \backslash B(g))=I\left(x g^{\delta}\right) \in \mathscr{F} .
$$

Hence $A \backslash B(g) \in \mathscr{F}$, a contradiction since $B(g) \in \mathscr{F}$ and $\mathscr{F}$ is an ultrafilter. Thus $g^{\nu} \notin D N / N$ if $g \in G \backslash H$ and so

$$
H^{\nu}=D N / N \cap G^{\nu} .
$$

Now by Theorem A, $D N / N$ ser $C / N$ and so

$$
H^{\nu}=D N / N \cap G^{\nu} \operatorname{ser} G^{\nu}
$$

But $\nu: G \rightarrow C / N$ is a monomorphism and so

$$
H \operatorname{ser} G \text {, }
$$

as required.

Proof of Corollary C. (i) and (ii). These are the special cases of Theorem B corresponding to the two special types of local systems for $(H, G)$ mentioned prior to its statement.

(iii) Let $\mathscr{S}$ be a set of serial subgroups of $G$ and $H=\langle\mathscr{P}\rangle$. Then the set comprising all the joins of finite subsets of $\mathscr{S}$ forms a local system for $H$. Further these joins are 
all serial in $G$ since the join of any two, and so any finite number of, serial subgroups of $G$ is serial in $G$. Now by (i) $H$ ser $G$. Hence $G \in \mathfrak{J}$.

(iv) Let $\left\{G_{\alpha} \mid \alpha \in A\right\}$ be a local system for $G$ and $G_{\alpha} \in \mathfrak{S}$ for all $\alpha \in A$. Suppose that $\mathscr{S}$ is a set of serial subgroups of $G$ and $H=\langle\mathscr{P}\rangle$. Let

$$
\mathscr{S}_{\alpha}=\left\{S \cap G_{\alpha} \mid S \in \mathscr{S}\right\} \text { and } H_{\alpha}=\left\langle\mathscr{P}_{\alpha}\right\rangle
$$

for all $\alpha \in A$. Since, for each $S \in \mathscr{Y}, S \cap G_{\alpha} \operatorname{ser} G_{\alpha} \in \mathfrak{I}$,

$$
H_{\alpha} \operatorname{ser} G_{\alpha} \text {. }
$$

Now let $F$ be any finite subset of $G$. Since $H=\langle\mathscr{S}\rangle$, there exists a finite subset $E$ of $\cup \mathscr{S}$ such that $F \cap H \subseteq\langle E\rangle$. Now since $\left\{G_{\alpha} \mid \alpha \in A\right\}$ is a local system for $G$, there exists $\alpha \in A$ such that $(F \backslash H) \cup E \subseteq G_{\alpha}$. Hence $E \subseteq \cup \mathscr{S}_{\alpha}$, so $F \cap H \subseteq\langle E\rangle \leqslant H_{\alpha}$ and so

$$
F \subseteq\left(G_{\alpha} \backslash H\right) \cup H_{\alpha}
$$

Thus we may apply Theorem B to deduce $H \operatorname{ser} G$. Whence it follows that $G \in \mathfrak{\Im}$.

\section{REFERENCES}

(1) I. N. HERSTEIN, Noncommutative rings (Mathematical Association of America, Menascha, Wis., 1968).

(2) K. K. Hickin and R. E. PHillips, On classes of groups defined by systems of subgroups, Arch. Math. (Basel) 24 (1973), 346-350.

(3) A. I. MAL'CEv, On a general method for obtaining local theorems in group theory, Ivanov. Gos. Ped. Inst. Učen. Zap. 1 (1941), 3-9.

(4) J. V. PETTY, Classes of groups defined by series or normal factor coverings, J. Algebra 40 (1976), 610-617.

(5) D. J. S. RoBINSON, Finiteness conditions and generalized soluble groups, part 2 (Springer-Verlag, Berlin, 1972).

DEPARTMENT OF MATHEMATICS, UNIVERSITY OF EDINBURGH, James Clerk MaXwell Building, EDINBURGH, EH9 3JZ. 